\title{
Single-incision mesh vs sacrospinous ligament fixation in posthysterectomy women at a three-year follow-up: a randomized trial
}

\author{
Galad $\mathbf{J}^{1,2}$, Papcun $\mathrm{P}^{3}$, Dudic $\mathrm{R}^{2}$, Urdzik $\mathrm{P}^{1,2}$ \\ Gynaecological Department, Gynpor, s.r.o, Sliac, Slovakia. peter.urdzik@upjs.sk
}

\begin{abstract}
OBJECTIVES: The primary clinical objective was to prospectively compare the effectiveness of the "single-incision MESH" technique versus sacrospinous ligament fixation (ACSSF) in correcting the defect of pelvic organ prolapse (POP) in the anterior and apical compartments, Their effectiveness was assessed at a 1-year/3-year follow-up (FU). METHODS: In the period of 2013-2015, we have randomized 146 women into two groups, namely 73 into ACSSF group and 73 into MESH group

RESULTS: At 1-year/3-year FU, we achieved an effectiveness of $92 \% / 87 \%$ in the point $\mathrm{Ba}(\leq-1 \mathrm{~cm})$ in the MESH group (48/52; 40/46) vs $70 \% / 66 \%$ in the ACSSF group $(35 / 50 ; 30 / 45) ;(p=0.005 / p=0.021)$. At 1 -year/3-year FU, in the area of point $\mathrm{C}(\leq-1 \mathrm{~cm})$, we achieved a $94 \% / 91 \%$ effectiveness in the MESH group (49/52; 42/46) vs $90 \% / 80 \%$ in the ACSSF group (45/50; 36/45); $(p=0.005 / p=0.192)$. In the MESH group, we observed a more frequent occurrence of "de novo" SUI (11\% vs $6 \%$ ) and a defect in the unoperated (posterior) compartment (18\% vs $8 \%$ ); the difference was not statistically significant. CONCLUSION: Our study showed that the vaginal synthetic mesh repair of POP did not improve women's outcomes in terms of effectiveness or adverse effects, while the patient satisfaction is the same as compared to that with sacrospinous ligament fixation at a 3-year FU. In gynecology, there are situations in which the comparison replaces the scientific solution (Tab. 5, Fig. 2, Ref. 50). Text in PDF www.elis.sk KEY WORDS: sacrospinous ligament fixation, quality of life index, synthetic mesh, randomized trial, prolapse.
\end{abstract}

\begin{abstract}
Abreviations: 1 y-FU - 1-year follow-up, 3 y-FU - 3-year followup, ACSSF - Anterior colporrhaphy with sacrospinous fixation, AHYE - Abdominal hysterectomy, BMI - Body mass index, FDA - Food and drug administration, FU - Follow-up, IQoL - Quality of life index, LAVH - Laparoscopic assisted vaginal hysterectomy, LCFA - Last carried forward analysis, OAB - Overactive bladder, OABI - Overactive bladder improvement, POP - Pelvic organ prolapsed, POP-Q - Pelvic organ prolapse quantification, RUTC - Relapse in untreated compartment, SI - Stress incontinence, TNO - Total number of operations, TVL - Total vaginal length, VAS - Visual analogue scale, VHYE - Vaginal hysterectomy
\end{abstract}

\section{Introduction}

We got access to material in form of polypropylene meshes (MESH), which demonstrated good biocompatibility and promised the possibility of reliable replacement of defective tissues (1). Unfortunately, it was shown that their implantation also comes

${ }^{1}$ Gynaecological Department, Gynpor, s.r.o, Sliac, Slovakia, ${ }^{2}$ Department of Gynaecology and Obstetrics, Safarik University in Kosice, Slovakia, and ${ }^{3}$ nnd Department of Gynecology and Obstetrics, Comenius University in Bratislava, Slovakia

Acknowledgement: This study was supported by the Scientific Grant Agency of the Ministry of Education of the Slovak Republic; Contract No. Grant VEGA 1/0873/18 and 1/0620/19. with certain long-term risks that we have not encountered in classical techniques (2-4). At present, not only we are in a period of searching for a prudent indication for their use (5), but also, in some countries, there has been a complete ban on the production and distribution of vaginal meshes used in POP surgical treatment and stressed incontinence $(6,7)$.

The aim of the randomized study was to compare the effectiveness of the correction of a defect in the anterior and apical compartments by means of the "single incision MESH" technique versus classical surgical technique, as well as patient satisfaction with procedures by using scoring systems in the scope of surgical treatment of pelvic organs in patients after hysterectomy.

\section{Materials and methods}

This was a prospective randomized study. We formed two parallel groups of women who were to undergo a transvaginal concomitant anterior/apical compartment repair using the single-incision synthetic MESH technique or classical anterior repair with sacrospinous ligament fixation technique (ACSSF). The randomisation was performed by using an envelope before the surgery.

\section{Inclusion criteria}

a) age over 18 years, b) pelvic organ prolapse quantified as $3 \mathrm{rd}$ degree in anterior compartment and $2 \mathrm{nd} / 3 \mathrm{rd}$ degree in apical 
compartment, c) history of performed hysterectomy, d) signed informed content.

\section{Exclusion criteria}

a) concomitant operation (posterior prolapse pelvic repair), b) uterus in situ, c) POP-Q $>3$.

\section{Statistical analysis - chart flow power analysis}

We planned to follow up 146 women in total ( 73 in each arm) to detect a difference in the primary clinical outcome POP-Q (Ba $\leq-1 \mathrm{~cm} ; \mathrm{C} \leq-1 \mathrm{~cm}-$ POP-Q) of $20 \%$ in favour of MESH group with $90 \%$ power and $\alpha=0.05$, where an efficacy of $70 \%$ was assumed with standard vaginal surgery method without the synthetic mesh. The sample size was increased to 174 women to allow for a potential $15 \%$ drop out.

In the period of 2013-2015, we identified 453 women with POP, of whom 223 were ineligible for the study, thus leaving us with 230 women eligible for randomisation, of whom however, 83 declined their participation. Finally, 146 women were randomised in the study. According to the selected operation technique, we prospectively, in form of a sealed envelope opened directly in the operation theatre, divided the 146 women into two groups based on whether the correction was to be performed with MESH "single incision" technique (73 women) or classic technique, i.e anterior colporrhaphy with sacrospinous ligament fixation (ACSSF; 73 women). Due to complications during anesthesia (diastolic blood pressure over $110 \mathrm{mmHg}$, allergic reaction to pharmacy, bronchospasm, etc.) the operation was not performed in 5 women ( $3 \mathrm{MESH} / 2 \mathrm{ACSSF}$ ) and other concomitant surgery had to be added in 14 women (6 MESH/8 ACSSF). Finally, the MESH surgery was performed in 64 women and ACSSF surgery in 63 women.

Figure 1 presents a detailed flowchart of patients randomized in the study.

\section{Preoperative measurements}

Medical history of each of the patients was obtained before the surgery, and each patient was physically examined in detail. For each patient in both groups, we determined the value of reference points $\mathrm{Aa}, \mathrm{Ba}, \mathrm{C}, \mathrm{Ap}, \mathrm{Bp}$, Gh, Gp and tvl with the degree of the defect determined according to the POP-Q classification (8). The preoperative urine culture from each patient was negative. A coughing test with repositioning of the prolapse was conducted in every patient for determining the occult stress incontinence.

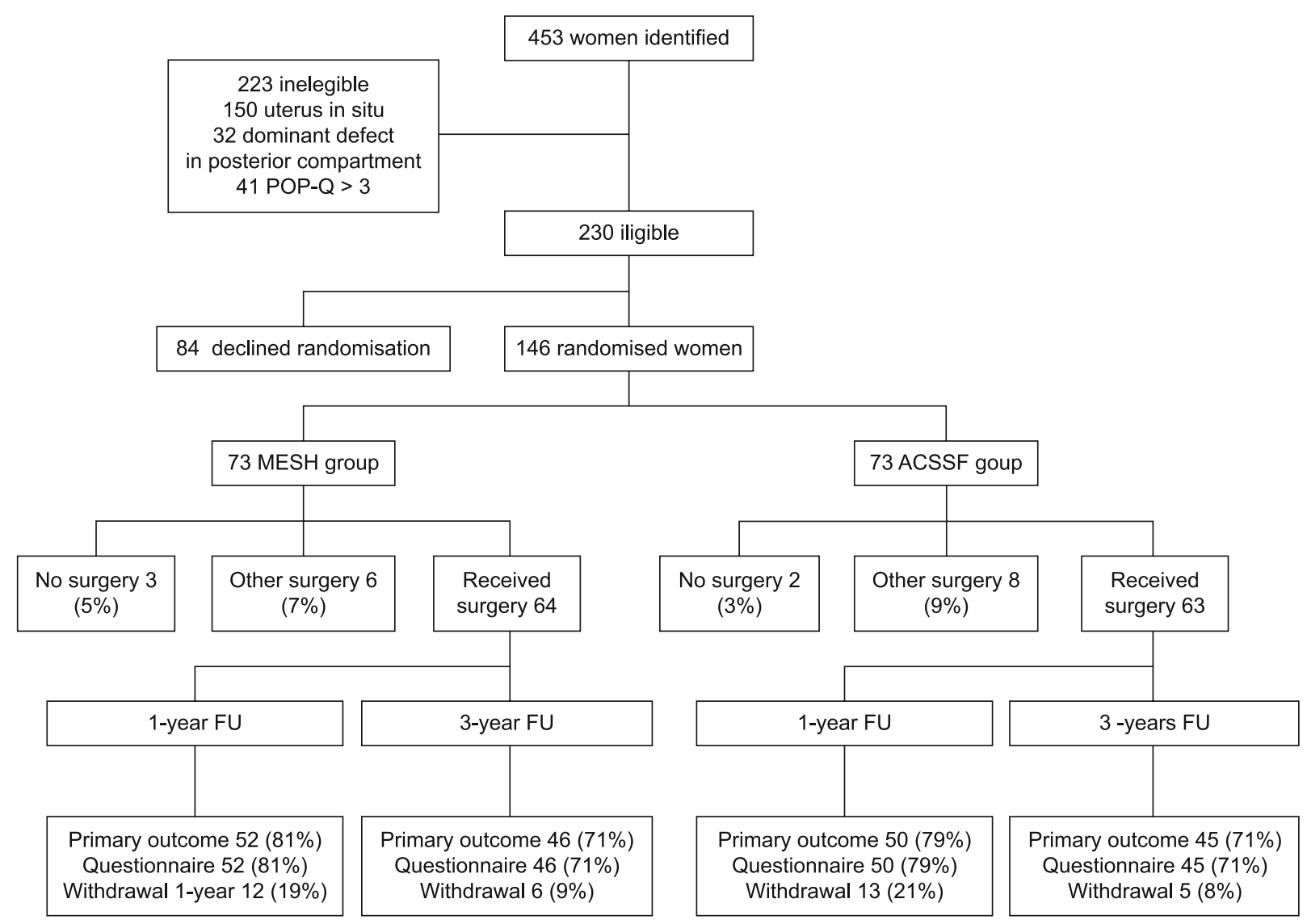

Fig. 1. Flowchart. 


\section{Postoperative measurements.}

We determined the degree of the defect according to the POP-Q upon admittance of each patient in both groups and then at 1-year and 3-year follow-ups. The correction was considered successful when the degree in the operated compartment was $\leq 1$ (point $\mathrm{Ba}$ $\leq-1 \mathrm{~cm}$; point $\mathrm{C} \leq-1 \mathrm{~cm}$ ) while a degree of $\geq 2$ in the operated compartment according to POP-Q was considered to be a recurrence of the defect. We compared the results achieved in MESH and ACSSF groups using the chi-square test, and determined the statistical significance while $\mathrm{p}<0.05$ was considered to be statistically significant.

\section{Surgical procedures}

Every patient received antibiotic prophylaxis with Cefazolin 1 g 30 minutes before surgery; The Foley catheter was left for 24 hours and Betadine flush was performed for a period of 4-6 weeks.

In the MESH group, in the scope of reconstruction of the combined defect in the anterior and medial compartments, we introduced the implants Elevate Anterior \& Apical or NUVIA SI anterior. The principle of this operation lies in the placement of the implant into the vesicovaginal space, and in direct fixation of the arms of the mesh to the sacrospinous ligament and obturator membrane using plastic anchors.

In the ACSSF group, in the scope of reconstruction of the combined defect in the anterior and medial compartments, we operated on patients with the technique of anterior colporrhaphy with sacrospinous ligament fixation. The principle of this operation is in the gathering of the vesicovaginal fascia and unilateral fixation of the vaginal stub to ligamentum sacrospinosum from the anterior colpotomy.

\section{Outcomes}

The primary clinical outcome in form of surgical efficacy of the compared methods was defined as a maximum leading edge of $<-1 \mathrm{~cm}$ (POP-Q) at 1-year and 3-year follow-ups. The secondary outcome in form of patient satisfaction and improvement with procedures was determined by using scoring systems in form of visual analogue scale (VAS) and index of quality of life. We also observed adverse effects and complications of surgery (exposure of synthetic MESH, dyspareunia, de novo overactive bladder, and stress urinary incontinence, etc). We also reported the recurrence of prolapse in untreated (posterior) compartment.

\section{Statistical analysis}

Quantitative data were expressed as average \pm standard deviation while qualitative data were expressed as percentage of the number of positive cases (n). The paired t-test was used for comparing quantitative parameters between individual groups. We examined the data for normal distribution and used Mann-Whitney test when normality was not met. The Chi-square test was used for comparing qualitative data between the groups. The paired t-test was used to compare the data in one group in different time horizons. The programs Microsoft Excel 365 ProPlus version 1811 and IBM SPSS Statistics version 20 were used for statistical evaluations. All statistical evaluations were performed at the level of statistical significance $\mathrm{p}<0.05$ (9).

\section{Ethics}

The study protocol was approved by decision No. 1/2012 of the Ethics Committee of the Gynpor General Hospital, Sliač, Slovak Republic. At the same time, the protocols and informed consent were in line with legislation of the Slovak Republic pursuant to Act No. 122/2013 Coll. and Act No. 84/2014 Coll. The study protocol was carefully explained to each patient, and each patient signed the informed consent on inclusion in the study.

\section{Results}

In the period of 2013-2015, we identified 453 women with POP, of whom 223 were ineligible for the study, thus leaving us with 230 women eligible for randomisation, of whom however, 83 declined their participation. Finally, 146 women were randomised in study. The surgery was performed in 64 women in the MESH group, and 63 women in the ACSSF group. Detailed analysis (surgery efficacy/questionnaire) was performed on the data from 102 women. We also performed an analysis with the inclusion of patients who failed to appear for the check-up or deliver the questionnaire. In Table 1, the patient baseline characteristics of the set are presented.

Table 2 presents an objective evaluation of points according to POP-Q classification before the operation and at 1-year and 3-year follow-ups.

\section{Primary outcome}

\section{Correction of defect in MESH group}

We assigned 52 women with a combined defect in the anterior (POP-Q - 3) and medial compartments (POP-Q 2-3) to the MESH group. The characteristics of the set are presented in Table 1. Hysterectomy was performed in 52 of the women (100\%) in the

Tab. 1 Patient baseline characteristics.

\begin{tabular}{lccc}
\hline Parameter & $\begin{array}{c}\text { MESH } \\
(\mathrm{n}=52)\end{array}$ & $\begin{array}{c}\text { ACSSF } \\
(\mathrm{n}=50)\end{array}$ & $\begin{array}{c}\text { t-test }^{\mathrm{a}} \text { / } \\
\text { chi-square test }^{\mathrm{b}}\end{array}$ \\
\hline Age & $63.1 \pm 8.7$ & $63.6 \pm 8.7$ & $0.745^{\mathrm{a}}$ \\
Parity & $2.6 \pm 1.1$ & $2.6 \pm 1.5$ & $0.947^{\mathrm{a}}$ \\
BMI & $27.4 \pm 3.9$ & $27.6 \pm 3.8$ & $0.729^{\mathrm{a}}$ \\
LAVH & $35 \%(18)$ & $36 \%(18)$ & $0.524^{\mathrm{b}}$ \\
VAHYE & $44 \%(23)$ & $34 \%(17)$ & $0.196^{\mathrm{b}}$ \\
AHYE & $21 \%(11)$ & $30 \%(15)$ & $0.213^{\mathrm{b}}$ \\
Sexual activity & $48 \%(25)$ & $50 \%(25)$ & $0.502^{\mathrm{b}}$ \\
OAB & $63 \%(33)$ & $56 \%(28)$ & $0.286^{\mathrm{b}}$ \\
SI in history & $21 \%(11)$ & $16 \%(8)$ & $0.340^{\mathrm{b}}$ \\
Operation time & $42 \pm 7 \mathrm{~min}$ & $35 \pm 6 \mathrm{~min}$ & $0.624^{(\mathrm{b})}$ \\
Blood loss $>300 \mathrm{ml}$ & $0 \%(0 / 52)$ & $0 \%(0 / 50)$ & - \\
PMR $>200 \mathrm{ml}$ & $2 \%(1 / 52)$ & $12 \%(6 / 50)$ & n.s. ${ }^{(\mathrm{b})}$ \\
Organ injury & $0 \%(0 / 52$ & $2 \%(1 / 50)$ & n.s. \\
\hline
\end{tabular}

BMI - body mass index, LAVH - laparoscopic assisted vaginal hysterectomy, VHYE - vaginal hysterectomy, AHYE - abdominal hysterectomy, OAB - overactive bladder, $\mathrm{SI}$ - stress incontinence, PMR - post-miction residuum, Chi-square test ${ }^{\mathrm{b}}$ for qualitative data $\mathrm{p}<0.05$, Paired $\mathrm{t}$-test $\mathrm{t}^{\mathrm{a}}$ for quantitative data $\mathrm{p}<0.05$ 
Tab. 2. Pelvic organ prolapse quantification measurement at preoperative and postoperative 1-year and 3-year follow-ups according to surgical method (5).

\begin{tabular}{|c|c|c|c|c|c|c|c|c|c|}
\hline & \multicolumn{3}{|c|}{ preoperative } & \multicolumn{3}{|c|}{ 1-year follow-up } & \multicolumn{3}{|c|}{ 3-year follow-up } \\
\hline & $\begin{array}{c}\text { MESH } \\
\mathrm{n}=64\end{array}$ & $\begin{array}{c}\text { ACSSF } \\
n=63\end{array}$ & $\mathrm{P}^{*}$ & $\begin{array}{c}\text { MESH } \\
n=52\end{array}$ & $\begin{array}{c}\text { ACSSF } \\
\mathrm{N}=50\end{array}$ & $\mathrm{P}^{*}$ & $\begin{array}{c}\text { MESH } \\
\mathrm{n}=48\end{array}$ & $\begin{array}{c}\text { ACSSF } \\
n=45\end{array}$ & $\mathrm{P}^{*}$ \\
\hline $\mathrm{Aa}$ & $2.34 \pm 0.6$ & $2.24 \pm 0.4$ & 0.346 & $-1.82 \pm 0.7$ & $-1.35 \pm 0.8$ & 0.0004 & $-1.70 \pm 0.7$ & $-1.33 \pm 1.0$ & 0.051 \\
\hline $\mathrm{Ba}$ & $2.91 \pm 0.7$ & $2.89 \pm 0.4$ & 0.897 & $-2.5 \pm 0.8$ & $-1.57 \pm 1.3$ & $<0.0001$ & $-2.27 \pm 0.9$ & $-1.57 \pm 1.4$ & 0.021 \\
\hline $\mathrm{C}$ & $0.63 \pm 1.4$ & $0.61 \pm 1.2$ & 0.803 & $-4.03 \pm 1.3$ & $-3.60 \pm 1.4$ & 0.457 & $-3.79 \pm 1.0$ & $-3.41 \pm 1.0$ & 0.045 \\
\hline Ap & $-1.57 \pm 0.7$ & $-1.59 \pm 0.6$ & 0.861 & $-1.53 \pm 1.3$ & $-1.48 \pm 1.1$ & 0.204 & $-1.33 \pm 1.6$ & $-1.68 \pm 1.1$ & 0.971 \\
\hline $\mathrm{Bp}$ & $-2.2 \pm 0.7$ & $-2.18 \pm 0.7$ & 0.830 & $-2.04 \pm 1.7$ & $-1.77 \pm 1.6$ & 0.028 & $-1.56 \pm 2.2$ & $-1.92 \pm 1.3$ & 0.229 \\
\hline TVL & $9.67 \pm 1.0$ & $9.38 \pm 0.8$ & 0.123 & $-1.11 \pm 1.2$ & $-1.01 \pm 1.2$ & 0.138 & $-1.10 \pm 1.2$ & $-1.00 \pm 0.9$ & 0.142 \\
\hline
\end{tabular}

$\mathrm{Aa}$ - anterior wall $3 \mathrm{~cm}$ from hymen; $\mathrm{Ap}$ - posterior wall $3 \mathrm{~cm}$ from hymen; $\mathrm{Ba}$ - anterior wall, most dependent par $(\mathrm{cm})$; Bp - posterior wall, most dependent par ( $\mathrm{cm})$; $\mathrm{C}$ - cervix or vaginal cuff $(\mathrm{cm}) ; \mathrm{TVL}=$ total vaginal length $(\mathrm{cm}) ;{ }^{*}$-value of Mann-Whitney test

Tab. 3. Surgical efficacy at 1-year and 3-year follow ups / last carried forward analysis according to surgical method (MESH vs ACSSF).

\begin{tabular}{|c|c|c|c|c|c|c|}
\hline & \multicolumn{3}{|c|}{ After 1 year } & \multicolumn{3}{|c|}{ After 3 years } \\
\hline & MESH & ACSSF & $\mathrm{p}$ & MESH & ACSSF & $\mathrm{p}$ \\
\hline$\overline{\mathrm{POP}-\mathrm{Q} \leq 1(\mathrm{Ba})}$ & $92 \%(48 / 52)$ & $70 \%(35 / 50)$ & 0.004 & $87 \%(40 / 46)$ & $66 \%(30 / 45)$ & 0.020 \\
\hline POP-Q $\leq 1(\mathrm{C})$ & $94 \%(49 / 52)$ & $90 \%(45 / 50)$ & 0.336 & $91 \%(42 / 46)$ & $80 \%(36 / 45)$ & 0.107 \\
\hline LCFA $-\mathrm{POP}-\mathrm{Q} \leq 1(\mathrm{Ba})$ & $75 \%(48 / 64)$ & $55 \%(35 / 63)$ & 0,017 & $63 \%(40 / 64)$ & $47 \%(30 / 63)$ & 0,066 \\
\hline LCFA - POP-Q $\leq 1$ (C) & $76 \%(49 / 64)$ & $71 \%(45 / 63)$ & 0,324 & $65 \%(42 / 64)$ & $57 \%(36 / 63)$ & 0,212 \\
\hline
\end{tabular}

Chí-square test $\mathrm{p}<0.05$, LCFA - last carried forward analysis

Tab. 4. Quality of life index at 1-year and 3-year follow-ups.

\begin{tabular}{|c|c|c|c|c|c|c|}
\hline & \multicolumn{3}{|c|}{ 1-year FU } & \multicolumn{3}{|c|}{ 3-year FU } \\
\hline & \multirow{2}{*}{ MESH } & \multirow{2}{*}{ ACSSF } & $\mathrm{P}$ & \multirow{2}{*}{ MESH } & \multirow{2}{*}{ ACSSF } & $\mathrm{P}$ \\
\hline & & & $\mathrm{t}$-test (t)/Fischer test (FT) & & & $\mathrm{t}$-test (t)/Fischer test (FT) \\
\hline Subjective & $6.63 \pm 2.25$ & $6.34 \pm 2.45$ & $0.529(\mathrm{t})$ & $5.98 \pm 2.23$ & $6.07 \pm 2.27$ & $0.852(\mathrm{t})$ \\
\hline Excellent & $44 \%(23 / 52)$ & $36 \%(18 / 50)$ & 0.777 (FT) & $27 \%(13 / 48)$ & $20 \%(9 / 45)$ & 0.898 (FT) \\
\hline Very good & $25 \%(13 / 52)$ & $26 \%(13 / 50)$ & & $33 \%(16 / 48)$ & $38 \%(17 / 45)$ & \\
\hline Good & $25 \%(13 / 52)$ & $28 \%(14 / 50)$ & & $32 \%(15 / 48)$ & $33 \%(15 / 45)$ & \\
\hline Poor & $6 \%(3 / 52)$ & $10 \%(5 / 50)$ & & $8 \%(4 / 48)$ & $9 \%(4 / 45)$ & \\
\hline
\end{tabular}

T-t-test, FT - Fischer test, FU- follow-up

past. The average operation time was $42( \pm 7)$ min. The control of patients was scheduled at regular intervals of 6 weeks, 6 months, 1 year and 3 years. In the MESH group, the effectiveness in the anterior compartment (point $\mathrm{Ba} \leq-1 \mathrm{~cm}$ ) at the 1-year follow up was $92 \%$ (48/52), while in the area of the medial compartment (point $\mathrm{C} \leq-1 \mathrm{~cm}$ ) it was $94 \%(49 / 52)$. At the 3-year follow-up in the latter group we achieved an effectiveness of $87 \%$ in the anterior compartment (point $\mathrm{Ba} ; 40 / 46$ ), and $91 \%$ in the medial compartment (point $\mathrm{C} ; 42 / 46$ ).

Correction of defect in group with classical ACSSF technique

We assigned 50 women with a combined defect in the anterior (POP-Q - 3) and medial compartments (POP-Q 1-2) to the ACSSF group. The characteristics of the set are presented in Table 1. Hysterectomy was performed on 50 of the women (100\%) in the past, and the average operation time was $36( \pm 6) \mathrm{min}$. At the 1-year follow-up, the effectiveness in the anterior compartment (point $\mathrm{Ba} \leq-1 \mathrm{~cm})$ was $70 \%(35 / 50)$, while that achieved in the area of the medial compartment (point $\mathrm{C} \leq-1 \mathrm{~cm}$ ) was $90 \%(45 / 50)$. At the 3-year follow-up, the effectiveness achieved in the anterior compartment was $66 \%$ (point $\mathrm{Ba} ; 30 / 45$ ), while that achieved in the area of the medial compartment (point C) was $80 \%(36 / 45)$.

Comparison of results from MESH and classic ACSSF technique groups

At the 1-year follow-up, the difference in the effectiveness in the anterior compartment (point $\mathrm{Ba}$ ) of $92 \%$ achieved in the MESH group (48/52) versus that of $70 \%(35 / 50)$ achieved in the ACSSF group was statistically significant $(p=0.005)$. In the area of the medial compartment (point $\mathrm{C}$ ), the difference between the effectiveness of $94 \%(49 / 52)$ achieved in the MESH group versus $90 \%(45 / 50)$ achieved in the ACSSF group was not statistically significant. At the 3-year follow-up, the difference in the effectiveness in the anterior compartment (point Ba) of $87 \%(40 / 46)$ achieved in the MESH group versus $66 \%(30 / 45)$ achieved in the ACSSF group was statistically significant $(p=0.021)$. In the area of the medial compartment (point $\mathrm{C}$ ), the difference in the effectiveness of $91 \%(42 / 46)$ achieved in the MESH group versus $80 \%(36 / 45)$ achieved in the ACSSF group was not statistically significant. We also performed an analysis inclusive of data 


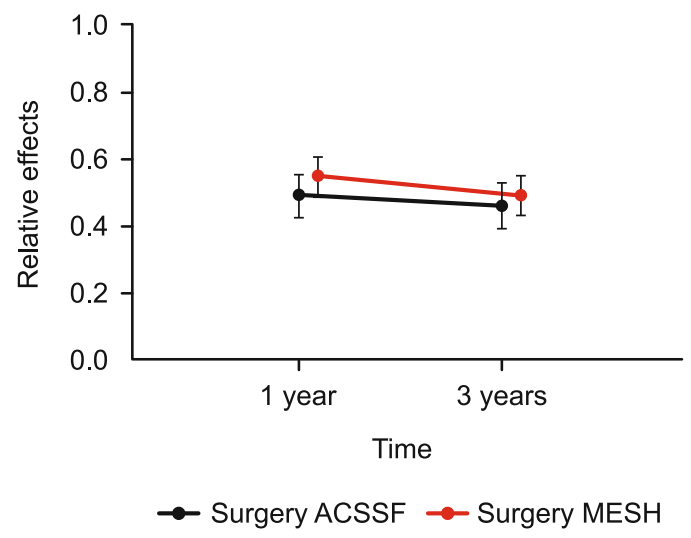

Fig. 2. Relative IQoL satisfaction.

from patients who failed to present for a check-up or deliver the questionnaire (Tab. 3).

\section{Secondary outcome - IQoL and VAS}

At the time of 1-year and 3-year follow-ups, we monitored also the satisfaction with the surgical solution. The patients evaluated their own state on the basis of IQoL and VAS 10. We evaluated the results by using the combination of unpaired t-test and chi-square test at the level of $\mathrm{p}<0.05$. At the one-year follow-up, $94 \%$ of patients in the MESH group (49/52) evaluated their own state as minimally good in comparison with $88 \%$ in the ACSSF group (44/50). The difference was not statistically significant. At the 3-year follow-up, $91 \%$ of patients in the MESH group (42/46) were satisfied versus $87 \%$ in the ACSSF group (39/45), and the difference was also not statistically significant. It can be stated that in both groups, the majority of patients were subjectively satisfied with the result of the operation and level of quality of their life (Tab. 4).

We also performed a longitudinal data analysis of IQoL with nonparametric longitudinal data analysis (9). We observed that there was a nonsignificant difference between MESH and ACSSF, both before and after the surgery $(p=0.769)$. IQoL, however, was significantly lower after surgery in both arms $(p<0.001)$ (Fig. 2).

\section{Complications with reconstructive POP operations with the use of MESH and ACSSF}

We observed also adverse effects and complications of surgery (exposure of synthetic MESH, dyspareunia, overactive bladder, etc.). We also reported a recurrence of prolapse in untreated (posterior) compartment (Tab. 5).

\section{Discussion}

We performed a randomized study on 146 women with a combined defect in anterior and apical compartments after hysterectomy.

Strong points of our study were a) randomisation, b) ensured uniformity of patients assigned to the set as well as that of the type and degree of defect c) uniformity of surgical procedures (every operation was performed by the same surgeon).

In our prospective study, we showed that the effectiveness of the correction of the anterior compartment determined at 1-year and 3-year follow-ups was higher in the MESH group (point $\mathrm{Ba}$ : $92 \%$ and $87 \%$, respectively) in comparison with ACSSF group (point Ba: $70 \%$ and $66 \%$, respectively). Our results are comparable with those of other studies comparing the effectiveness of correction by use of the synthetic material Elevate anterior or NUVIA SI anterior (10-13).

On the other hand, when we compare the 3-year follow-up results in the medial compartment achieved with MESH (point C: 91\%) vs ACSSF (point C: $80 \%$ ), the difference is not statistically significant, and the study has shown that the repair of prolapse with the synthetic mesh confers no anatomical benefits to women in the short term (14-16).

However, at a remove of years, we have to state that in line with other studies (17-21), there was a higher risk of a defect occurring de novo in the so-called unoperated compartment (in

Tab. 5. Postoperative complications at 1-year and 3-year follow-ups according to surgical method (MESH vs ACSSF.)

\begin{tabular}{|c|c|c|c|c|c|c|}
\hline & \multicolumn{3}{|c|}{ 1-year FU } & \multicolumn{3}{|c|}{ 3-year FU } \\
\hline & MESH n=52 & ACSSF $\mathrm{n}=50$ & $\mathrm{p}$ & $\mathrm{MESH} n=46$ & ACSSF $n=45$ & $\mathrm{p}$ \\
\hline Mesh exposure & $6 \%(3)$ & $1 \%(1)$ & 0.618 & $8 \%(4)$ & $0 \%$ & 0.117 \\
\hline Dyspareunia & $10 \%(2 / 21)$ & $9 \%(2 / 23)$ & 1 & $14 \%(3 / 21)$ & $9 \%(2 / 23)$ & 0.658 \\
\hline OABI & $73 \%(16 / 22)$ & $62 \%(15 / 24)$ & 0.539 & - & - & - \\
\hline OAB de novo & $6 \%(3)$ & $8 \%(4)$ & 0.713 & - & - & - \\
\hline SI de novo & $11 \%(6)$ & $6 \%(3)$ & 0.488 & - & - & - \\
\hline RUTC & $10 \%(5)$ & $6 \%(3)$ & 0.716 & $18 \%(9)$ & $8 \%(4)$ & 0.230 \\
\hline SI surgery & $6 \%(3)$ & $4 \%(2)$ & 1 & - & - & \\
\hline RUTC & $4 \%(2)$ & $2 \%(1)$ & 1 & $4 \%(2)$ & $2 \%(1)$ & 1 \\
\hline RUTC surgery & 0 & $4 \%(2)$ & 0.238 & $2 \%(1)$ & $6 \%(3)$ & 0,361 \\
\hline Exposure Surgery & $4 \%(2)$ & 0 & 0.495 & $2 \%(1)$ & 0 & 1 \\
\hline TNO & $14 \%(7)$ & $10 \%(6)$ & 1 & $8 \%(4)$ & $8 \%(4)$ & 1 \\
\hline
\end{tabular}


range of $5-15 \%$ ) at 1 -year and 3-year follow-ups in the MESH group $(5 / 10 \%$ and $9 / 18 \%$, respectively) in comparison with the ACSSF group (3/6 \% and $4 / 8 \%$, respectively). We ascribe these conclusions to the fact that in case that synthetic material is used, the strength of fixation in the apex of the vagina exerts excessive pressure in the unsupported compartment, thus enhancing the formation of a new defect.

In both groups, at 1-year and 3-year follow-ups, there was observed a lengthening of the vagina as compared to its state before the operation, which may have a positive effect on the quality of sexual life $(22,23,24)$. Another monitored modality was the occurrence of complications, which in case that synthetic material is used is one of the main reasons for reclassifying the method from category II to category III, and each patient must be instructed especially regarding the risk of later complications in form of protrusion, origin of chronic pelvic pain, etc. $(5,25)$.

In the MESH group, protrusions occurred, namely 3 (6\%) at 1 -year follow-up and $4(8 \%)$ at 3 -year follow-up. These results are comparable with the results of other studies that used synthetic material $(10,11,26,27)$. In both sets, there was a conformity with other studies (28-30), namely in the significant disappearance of OAB symptoms (MESH $73 \%$ vs ASCCF $62 \%$ ); the difference, however, was not statistically significant. Similarly, we also found a lower risk of $\mathrm{OAB}$ occurring de novo in the MESH group (6\%) as compared to ACSSF group ( $8 \%$ ), and reversely, the occurrence of SI "de novo" at 1-year follow-up was higher in the MESH group (11\%) as compared to the ACSSF group (6\%); the difference, however, was not statistically significant. Our findings were similar to those of other studies dealing with the association of the combined defect (anterior/apical) with increased odds of SUI surgery in the future $(15,31-33)$.

Likewise, we did not find any difference in the duration of hospitalization in the monitored set. Nearly all the patients were released within 48 hours after the operation.

No major loss of blood (higher than $300 \mathrm{ml}$ ) occurred in either group and the surrounding organs were injured only once ( $1 \mathrm{x}$ urinary bladder in the ACSSF group). Therefore, in line with other studies, we consider the single-incision mesh technique to be equally safe and at the same time more effective in comparison with classic techniques $(11,26,34-36)$.

Our study is in line with other studies $(15,37-39)$ in showing that at 1-year and 3-year follow-ups, both groups showed a significantly improved quality of life in comparison with that in the preoperative period. We evaluated the index of improvement in the quality of life using VAS where patients from MESH group and ACSSF group assessed their own state as improved, namely in $94 \%$ and $91 \%$ at one-year follow up and 3-year follow up, respectively in the MESH group, and in $88 \%$ and $87 \%$ at one-year follow-up and 3-year follow-up, respectively in the ACSSF group. Our findings were in contrast with other studies that showed that many women reported symptoms that negatively affected their quality of life index (40-43).

In 2013, a register of synthetic implants was established in the Slovak Republic (44). The total number of operations with the use of synthetic material remains stable. However, there is an increase in the number of laparoscopic/abdominal sacrocolpopexy operations which are becoming the "gold standard" for vaginal prolapse (45-50).

\section{Learning points}

Our study showed that the vaginal synthetic mesh repair of the combined defect in anterior and apical compartments in women after hysterectomy did not improve their outcomes in terms of effectiveness, incidence of SI de novo, risk of relapse in the untreated compartment, and numbers of reoperation in the short term.

Despite the results of other studies, the quality of life index in both groups in our study (mesh/classical technique) is evaluated as an improvement.

The strong point of our study is that all patients underwent the surgery conducted at the same workplace and by the same experienced urogynecologist with many years of experience in the given field.

In the future, it will be essential to conduct additional studies with a larger number of respondents (multicentre, prospective) over longer time periods, as recommended by IUGA in 2016. However, in context of current changes $(5,25)$ and announcements of individual professional societies, it is still questionable whether it would be possible to carry out some form of prospective study in the future.

\section{References}

1. Winters JC, Fitzgerald MP, Barber MD. The use of synthetic mesh for female pelvic reconstructive surgery. BJU Int 2006; 98 (1): 70-76.

2. Kontogiannis S, Goulimi E, Giannitsas K. Reasons for and Against Use of Non-absorbable, Synthetis Mesh During pelvic Organe Prolapse Repair, According to the Prolapsed Compartment. Adv Ther 2017; 33 (12): 2139-2149.

3. Li YL, Chang YW, Yang TH, Wu LY, Chuang FC, Kung FT, Huang KH. Mesh related complications in single-incision transvaginal mesh (TVM) and laparoscopic abdominal sacrocolpexy (LASC). Taiwan J Obstet Gynecol 2020; 59 (1): 43-50. DOI: 10.1016/j.tjog.2019.11.06

4. Sohlberg EM, Dallas KB, Weeks BT, Elliott CS, Rogo-Gupta L. Reoperation rates for pelvic organ prolapse repairs with biologic and synthetic grafts in a large population-based cohort. Int Urogynecol J $2020 \mathrm{Feb} ; 31$ (2): 291-301. DOI: 10.1007/s00192-019-04035-3.

5. Food and Drug Administration, HHS. Obstetrical and Gynecological Devices; Reclassification of Surgical Mesh for Transvaginal Pelvic Organe Prolapse Repair; Final order. Fed Regist 2016; 81 (2): 353-361.

6. www.sfchronicle.com/opinion/openforum/article/Australia-andBritain-have-stopped-vaginal-mesh-13315968.php.

7. Fekete Z, Korosi S, Németh G. (Vaginal mesh operations in the urogynecological practice after the FDA warnings. Use or not to use mesh?) Orv Hetil 2018; 159 (10): 397-404. DOI: 10.1556/650.2018.30963.

8. Bump RC, Mattiasson A, Bo K, Brubaker LP, DeLancey JO, Klarskov PE. The standardization of terminology of female pelvic organe prolapse and pelvic floor dysfunction. Obstet Gynecol 1996; 176: 10-17.

9. Brunner E, Puri ML. Nonparametric Methods in Factorial Designs. Statistical Papers 2001; 42: 1-52. 
$640-647$

10. Castelanni D, Galica V, Saldutto P, Galatioto GP, Vicentini C. Efficasy and Safety of Elevate system on apical and anterior compartment prolapse repair with personal technique modification. Int Braz Urol 2017; 43 (6): 15-1121.

11. Lo TS, Al-Karabsheh AM, Tan YL, Pue LB, Hsieh WC, Uy-Patrimonio CM. Single insicision anterior apical mesh and sacrospinous ligament fixation in pelvic prolapse surgery at 36 month follow-up. Thaiwanese J Obstet Gynecol 2017; 6: 794-800.

12. Lin KL, Tang FH, Chou SH, Loo ZX, Liu YY, Juan YS, Long CY. Evaluation of single-incision apical vaginal suspension for treatment of pelvic organ prolapse. Eur J Obstet Gynecol Reprod Biol 2020; 247: 198-202. DOI: 10.1016/j.ojogrb.2020.02.013.

13. Slade E, Daly C, Mavranezouli I, Dias S, Kearney R, Hasler E, Carter $\mathbf{P}$ et al. Primary surgical management of anterior pelvic organ prolapse: a systematic review, network meta-analysis and cost-effectiveness analysis. BJOG 2020; 127 (1): 18-26. DOI: 10.1111/1471-0528. 15959.

14. Maher C, Feiner B, Baessler K, Schmid C. Surgical management of pelvic organe prolapse in women. Cochrane Database Syst Rev 2013; 4:CD004014

15. Glazener CM, Breeman S, Elders A et al. Mesh, graft, or standard repair for women having primary transvaginal anterior or posterior compartment prolapse surgery: two parallel-group, multicentre, randomized, controlled trials (PROSPECT). Lancet 2017; 389 (10067): 381-392.

16. Menefee S, Richter HE, Myers D, Weidner A, Moalli P, Harvie H, Rahn D et al. Design of a 3-Arm Randomized Trial for Posthysterectomy Vault Prolapse Involving Sacral Colpopexy, Transvaginal Mesh, and Native Tissue Apical Repair: The Apical Suyspension Repair for Vault Prolapse in a Three-Arm Randomizeed Trial. Female Pelvic Med reconstruct Surg 2019: 19. DOI: $10.1097 /$ SPV.0000000000000803.

17. Clark AL, Gregory T, Smith V J et al. Epidemiologic evaluation of reoperation for surgically treated pelvic organ prolapse and urinary incontinence. Am J Obstet Gynecol 2003; 5: 1261-1267.

18. Van Raalte HM, Lucente VR, Molden SM. One-year anatomic and quality-of-life outcomes after Prolift procedures for treatment of posthysterectomy prolapse. Am J Obstet Gynecol 2008; 6: 691-696.

19. Withagen MI, Vierhout ME, Milani AL. Does trocar-guided tensionfree vaginal mesh (Prolift) repair provoke prolapse of the unaffected compartments ? Int Urogynecol Floor Dysf 2010; 3: 271-278.

20. Urdzík P, Galad J, Ostró A. (Risk of the prolapse "de novo" in primary unaffected compartment by using the synthetic mesh in the surgery treatment of the pelvic organ prolapse.) Ces Gynekol 2011; 76: $321-326$

21. Gautier A, Ferry P, Bertherat P, De Tayrac R, Fernandez H. Transvaginal treatment of anterior and apical genital prolapse using Restorelle ${ }^{\circledR}$ direct fix ${ }^{\mathrm{TM}}$ : An observational study of medium-term complications and outcomes. J Gynecol Obstet Hum Reprod 2020; 49 (2): 101674. DOI: 10.1016/j.ogoh.2019.101674.

22. Ercan Ö, Özer A, Köstü B, Bakak M, Kiran G, Avci F. Comparison of postoperative vaginal lenght and sexual function after abdominal, vaginal and laparoscopic hysterectomy. Int J Gynaecol Obstet 2016; 132 (1): $39-41$.

23. Achermann APP, Brazäo ÉS Júnior, Riccetto CLZ, Palma PCR. Sacrospinous hysteropexy with a low weight transvaginal polypropylene mesh for treatment of complete uterovaginal eversion. Int Braz J Urol 2019; 45 (4): 856-857. DOI: 10.1590/S1677-5538.IBJU.2018.0555.
24. Juliato CRT, Santos-Junior LC, de Castro EB, Dertkigil SS, Brito LGO. Vaginal axis after abdominal sacrocolpopexy versus vaginal sacrospinous fixation- a randomized trial. Neurol Urodyn 2019; 38 (4): 1142-1151. DOI: 10.1002/nau.23970.

25. NICE Guidance - Urinary incontinence and pelvic organ prolapse in women: management: (C) MICE (2019) Urinary incontinence and pelvic organ prolapse in women:management. (No authors listed) BJU Int 2019; 123 (5): 777-803. DOI: 10.1111/bju. 14763.

26. Buca DIP, Liberati M, Falo $\mathbf{E}$ et al. Long-term outcome after surgical repair of pelvic organ prolapse with Elevate Prolapse Repair system. J Obstet Gynaecol 2018; 38 (6): 854-859.

27. Meriwether KV, Antosh DD, Olivera CK, Kim-Fine S, Balk EM, Murphy M, Grimes VCL et al. Uterine preservation vs hysterectomy in pelvic organe prolapse surgery: a systematic review with meta-analysis and clinical practice guidelines. Am J Obstet Gynecol 2018; 219 (2): 129-146. e2. DOI: 10.1016/.ajog.2018.01.018.

28. Foster RT, Barber MD, Paraiso MF et al. A prospective assessment of overactive bladder symptoms in a cohort of elderly women who underwent transvaginal surgery for advanced pelvic organe prolapse. Am J Obstet Gynecol 2007; 82 (1): 1-4.

29. Rogowski A, Kluz T, Szafarowska $M$ et al. Efficacy and safety of the Calistar and Elevate anterior vaginal mesh procedures. Eur J Obstet Gynecol Reprod Biol 2019; 239: 30-34.

30. de Castro EB, Brito LGO, Juliato CRT. Vaginal hysterectomy with bilateral sacrospinous fixation plus anterior mesh versus abdominal sacrocervicopexy for the treatment of primary apical prolapse in postmenopausal women: a randomized controlled study. Int Urogynecol J 2020; 31 (2): 365-372. DOI: 10.1007/s00192-019-03948-3.

31. Maher C, Feiner B, Baessler K, Christmann-Schmid C, Haya N, Brown J. Surgery for women with anterior comapartment prolapse. Cochrane Database Syst Rev 2016 30; 11:CD004014.

32. Syan R, Dallas KB, Sohlberg E, Rogo-Gupta L, Elliott CS, Enemchukwu EA. Rates and Risk Factors for Future Stress Urinary Incontinence Surgery after Pelvic Organe Prolapse Repair in a Large Population-based Cohort in California. Urology 2019; 123: 81-86.

33. Bastani P, Hajebrahimi S, Mallah F, Chaichi P, Sadeghi Ghiasi F. Long-term Outcome of Synthetic Mesh Use in Iranian Women with Genital Prolapse. Urol J 2020; 17 (1): 73-77. DOI: 10.22037/uj.v0i0.4866.

34. Hsieh HY, Tsai CP, Liu CK, Shen PS, Hung YC, Hung MJ. Factors that affect outcomes of prolapse repair using single-incision vaginal mesh procedures. Neurol Urodyn 2018; 37 (1): 298-306.

35. Gágyor D, Pilka R, Kudela M, Dzvinčuk P, Ondrová D, Benická A. Sakrospinous fixation sec. Miyazaki - complications and long-term results. Ces Gynekol 2019; 84 (2): 105-110.

36. Carter P, Fou L, Whiter F, Delgado Nunes V, Hasler E, Austin C, Ward $\mathrm{K}$ et al. Management of mesh complications following surgery for stress urinary incontinence and pelvic organ prolapse: a systematic review. BJOG 2020; 127 (1): 28-35. DOI: 10.1111/1471-0528. 15958.

37. Gonocruz SG, Hayashi T, Tokiwa S, Sawada Y, Okada Y, Yoshio Y, Krisna R et al. Transvaginal surgery using self.cut mesh for pelvic organ prolapse: 3-year clinical outcome. Int J Urol 2019; 26 (7): 731-736. DOI: $10.1111 /$ iju. 13984 .

38. Cadenbach-Blome T, Grebe M, Mengel M, Pauli F, Greser A, Funfgeld C. Significant Improvement in Quality of Life. Positive Effect on Sexuality, Lasting Reconstructive Result and Low rate of Complications 
Following cystocele Correction Using Lightweight, Large-Pore, Titanised Polypropylene Mesh: Final Results of a National, Multicentre Observation Study. Geburtshilfe Frauenhekild 2019; 79 (9): 959-968. DOI: 10.1055/a0984-6614.

39. da Silveira SDRB, Auge AP, Jarmy-Dibella ZI, Margarido PF, Carramao $\mathrm{S}$, Alves Rodrigues $\mathrm{C}$, Doumouchtsis $\mathrm{SK}$ et al. A multicentre, randomized trial comparing pelvic organe prolapse surgical treatment with native tissue and synthetic mesh: A 5.year follow-up study. Neurol Urodyn 2020; 39 (3): 1002-1011. DOI: 10.1002/nau.24323.

40. Milani R, Salvatore S, Soligo M, Pifarotti P, Meschia M, Cortese M. Functional and anatomical outcome after anterior and posterior vaginal prolapse repair with prolene mesh. BJOG 2004; 112 (1): 107-111.

41. Hansen BL, Dunn GE, Norton P, Hsu Y, Nygaard I. Long-term follow-up of treatment for synthetic mesh complications. Female Pelvic Med Reconstr Surg 2014; 20 (3): 126-130.

42. Dunn GE, Hansen BL, Egger MJ et al. Changed women: the longterm impact of vaginal mesh complications. Female Pelvic Med Reconstr Surg 2014; 20 (3): 131-136.

43. Tibi B, Vincens E, Durand M, Bentellis I, Salet-Lizee D, Kane A, Gadonneix P et al. Comparison of different surgical techniques for pelvic floor repair in elderly women: a multi-institutional study. Arch Gynecol Obstet 2019; 299 (4): 1007-1013. DOI: 10.1007/s00404-019-05076-1.

44. www.urogynekologia.sk/gynstat/login.php.
45. Maher CF, Feiner B, DeCuyper EM, Nichols CJ, Hickey KV, O'Rourke P. Laparoscopic sacral colpopexy versus total vaginal mesh for vaginal vault prolapse: a randomized trial. Am J Obstet Gynecol 2011; 204 (4): 360.e1-7.

46. Trochez RD, Lane S, Duckett J. The use of synthetic mesh for vaginal prolapse in the UK: a review of cases submitted to the British Society of Urogynaecology Database. Int Urogynecol J 2018.

47. Liang XZ, Xu LZ, Chen LQ, Wang S, Lin XT, Zhang XW. (Mid-term efficacy of laparoscopic sacral colpopexy of combined transabdominaltransvaginal approach in the treatment of stage IV pelvic organ prolapse). Zhonghua Fu Chan Ke Za Zhi 2019; 54 (3): 160-165. DOI: 10.3760/ cma.j.jssn.0529-5x.2019.03.004

48. Wei D, Wang P, Niu X, Zhao X. Comparison between laparoscopic uterus/sacrocolpopexy and total pelvic floor reconstruction with vaginal mesh for the treatment of pelvic organ prolapse. J Obstet Gynaecol Res 2019; 45 (4): 915-992. DOI: 10.1111/jog. 13908.

49. Smazinka M, Kalis V, Havir M, Havelkova L, Ismail KM, Rusavy Z. Obesity and its long-term impact on sacrocolpopexy key outcomes (OBELISK). Int Urogynecol J 2019. DOI: 10.1007/s00192-019-04076-8.

50. Pacquée S, Nawapun K, Claerhout F, Werbrouck E, Veldman J, D'hoore A, Wyndaele J et al. Long-Term Assessment of a Prospective Cohort of Patients Undergoing Laparoscopic Sacrocolpexy. Obstet Gynecol 2019; 134 (2): 323-332. DOI: 10.1097/AOG.00000000000003380.

Received April 12, 2020. Accepted May 12, 2020. 